\title{
POLÍticas ACTIVAS DE EMPLEO JUVENIL EN ECUADOR
}

\author{
ELISA LANAS MEDINA ${ }^{1}$
}

\section{RESUMEN}

El artículo presenta un estudio crítico sobre las políticas activas de empleo juvenil aplicadas en Ecuador por el actual gobierno, con especial énfasis en las incorporadas en la Ley Orgánica para la Promoción del Trabajo Juvenil, regulación excepcional de la jornada de trabajo, cesantía y seguro de desempleo. El objetivo de este estudio es comparar la política pública del gobierno en esta materia frente a los actuales planteamientos que organismos internacionales especializados proponen para enfrentar el alarmante desempleo de los jóvenes en todo el mundo. Se espera generar un debate informado sobre la pertinencia de dichas políticas y plantear la posibilidad de ajuste de los mecanismos propuestos en la norma.

PAlABRAS ClAVE: Política pública, políticas activas, políticas pasivas, empleo juvenil.

1 Docente a tiempo completo de la Universidad Andina Simón Bolívar 


\section{ABSTRACT}

This article offers a critical analysis of the existing youth employment policies implemented by the current Ecuadorian government, with special emphasis to those included in the Substantive Law on Youth Employment, Exceptional Regulation of Working Hours, and Unemployment Insurance. The goal of this study is to contrast the government's public policy on this matter with the approach that specialized international organisms propose to face the alarming youth unemployment rates across the world. This documents aims to generate an informed discussion over the relevance of said policies, and to explore the possibility to adjust the mechanisms proposed in the law.

\section{KEY WORDS:}

Public policy, active policies, passive policies, youth employment. 


\section{INTRODUCCIÓN}

La Constitución Política del Ecuador, reconoce todas las modalidades de trabajo, incluidas las de auto sustento y cuidado humano, y a sus actores como entes productivos ${ }^{2}$, no obstante, mantiene el énfasis en la importancia que el trabajo subordinado tiene, por seguir siendo el mayor espacio de absorción de demandantes de trabajo, y lo expresa destinando la sección octava del Título II, que trata sobre los derechos; para mostrar la relevancia que el trabajo tiene, como "un derecho y un deber social, y un derecho económico, fuente de realización personal y base de la economía", debiendo el Estado garantizar "a las personas trabajadoras el pleno respeto a su dignidad, una vida decorosa, remuneraciones y retribuciones justas y el desempeño de un trabajo saludable y libremente escogido o aceptado"4; principios que son desarrollados en la sección tercera del Capítulo Sexto del Título Sexto de la misma Carta Magna, que aborda el Régimen de Desarrollo.

Por su parte, el segundo inciso del artículo 39 de la Constitución ecuatoriana, prevé el reconocimiento que hace el Estado a los jóvenes como actores estratégicos del desarrollo del país, garantizándoles, además de la educación, salud, vivienda, recreación, deporte, tiempo libre, libertad de expresión y asociación, una especial atención para que su incorporación al trabajo sea en condiciones justas y dignas, con énfasis en la capacitación, la garantía de acceso al primerempleo y la promoción

2 Art. 325 de la Constitución Política de la República del Ecuador (en adelante $\mathrm{CE})$.

3 Art. $33 \mathrm{CE}$.

4 Ibíd. 
de sus habilidades de emprendimiento. Esta disposición se ve complementada por el primer inciso del artículo $329 \mathrm{CE}$, que enfatiza el derecho de los jóvenes a ser sujetos activos en la producción, así como en las labores de autosustento, cuidado familiar e iniciativas comunitarias, debiendo el Estado impulsar condiciones y oportunidades con ese fin.

Esto tiene gran significado en momentos como el actual, en que la economía ecuatoriana, al igual que las economías de todos los países de la región, enfrenta una desaceleración en su crecimiento. Lo que incide directamente en la vida y el bienestar de la población, que según investigaciones de CEPAL y OIT, en el contexto macroeconómico y laboral actual, podría provocar que en muchos países surja una mayor cantidad de empleos informales, para compensar parcialmente la ausencia de oportunidades de empleo productivo y de buena calidad ${ }^{5}$.

En este contexto, la situación de empleo de los jóvenes es aún más preocupante, ya que este colectivo tiene mayores dificultades para acceder a trabajos formales, debido a factores como la falta de experiencia, que es un elemento determinante a la hora de contratar trabajadores. Esta situación es aún peor para las mujeres y se ensaña con quienes no cuentan con niveles aceptables de escolarización.

Frente a esta realidad, el gobierno ecuatoriano ha emprendido varias reformas al Código del Trabajo y otros cuerpos normativos vinculados a la relación laboral, dos de ellos de importante

5 OIT CEPAL, «Coyuntura Laboral en América Latina y el Caribe. La evolución del empleo en las empresas de menor tamaño entre 2003 y 2013: mejoras y desafíos», octubre de 2015. 
magnitud. Estas reformas se han caracterizado por una cierta improvisación, traducida en una procesión acelerada de reformas laborales, sin una aparente claridad de objetivos a largo plazo, tal vez por la urgencia de enfrentar una tendencia claramente negativa en los niveles de empleo, derivada de una crisis económica que el gobierno se ha negado a aceptar pero que se ha instalado con toda su fuerza en el país y cuyas consecuencias se notan especialmente en los niveles de desempleo y subempleo, actualmente similares a los que el país vivió en el año 2007.

El presente estudio se centra en las políticas públicas activas de empleo juvenil, previstas especialmente en la Ley Orgánica para la Promoción del Trabajo Juvenil, regulación excepcional de la jornada de trabajo, cesantía y seguro de desempleo, para analizar su eficacia a la hora de lograr una mayor y mejor incorporación de los jóvenes al mercado laboral ecuatoriano.

\section{POLÍTICAS ACTIVAS Y PASIVAS DE EMPLEO}

La política pública es una cadena de decisiones y acciones, tomadas por actores, principalmente públicos y eventualmente privados, que persiguen la solución concreta de un problema definido como colectivo, es ineludible referirse a los grupos sociales que dan lugar al problema, conocidos como gruposobjetivo, así como al interés de los grupos que sienten los efectos del problema, que son los beneficiarios finales ${ }^{6}$.

6 Knoepfel Larrue, CoRinne Peter y otros, «Hacia un modelo de análisis de políticas públicas operativo. Un enfoque basado en los actores, sus recursos y las instituciones», Ciencia Política, Perspectivas teóricas, No 3 (junio de 2007). 
Dentro de estas, las políticas de empleo se presentan como ámbitos de intervención directa sobre el mercado de trabajo, sobre la oferta de trabajo (los demandantes de empleo) y sobre la demanda de trabajo (los empleadores); y su objetivo primordial es alcanzar un equilibrio entre la oferta y la demanda de trabajo, en sus aspectos cuantitativos y cualitativos, así como la protección de las situaciones de desempleo ${ }^{7}$.

Efectivamente, como complemento a otras intervenciones más macroeconómicas por parte del Estado, las políticas de empleo suponen una intervención directa que pretende el ajuste a corto plazo en el mercado de trabajo, por vía de reducir el porcentaje de desempleados y de empleados precarios, ofreciendo condiciones para que todos aquellos en edad de trabajar y con la disposición de hacerlo, puedan incorporarse al mercado de trabajo en condiciones adecuadas.

Una primera clasificación de las políticas de empleo distingue entre políticas activas y pasivas. Las políticas pasivas están destinadas a sostener la renta de los desempleados, por vía de garantizar un cierto nivel de ingresos para estas personas. "Tienen un carácter compensador frente a los efectos del mercado de trabajo". Se dirigen a los efectos del desempleo y no a sus causas. No afectan a la demanda ni oferta de empleo, únicamente a la renta del individuo perceptor.

7 Ramos Martín, Francisco, «Políticas de empleo», en Políticas sociolaborales, Economía y Empresa 17 (Barcelona: Editorial UOC, 2004).

8 Cabeza Pereiro, Jaime y Cardona Rubert, María B., «Políticas de empleo (I)», en Políticas sociolaborales, Tratados y manuales (Navarra: Thomson Reuters, 2014), p. 264. 
Mientras las políticas pasivas se dirigen exclusivamente a las personas desempleadas, las políticas activas tienen un espectro potencialmente más amplio, el de las personas desocupadas, con el fin de favorecer su inserción laboral y el de las ocupadas, para contribuir al mantenimiento de su empleo 9 .

\section{POLÍTICAS PASIVAS DE EMPLEO}

Por su propia estructura, las políticas pasivas, al tratarse de transferencias, suponen un mayor gasto para el Estado, y fueron la primera respuesta de los gobiernos al fenómeno del desempleo, ya que se vio que la garantía de rentas que las políticas pasivas suponen, permitía al trabajador desempleado tener unos ingresos garantizados mientras buscaba un nuevo empleo ${ }^{10}$.

Entre las políticas pasivas, las más importantes, sin duda alguna, son las prestaciones por desempleo, que pueden subdividirse en dos niveles ${ }^{11}$ :

Nivel contributivo. La prestación por desempleo es una prestación sustitutiva de los salarios que se abonan en función del tiempo y cuantía de la cotización. Se trata, propiamente, de un seguro de desempleo.

Nivel asistencial. Subsidio por desempleo que se abona a los desempleados que han agotado la prestación contributiva, o en

9 Cabeza Pereiro, Jaime y Cardona Rubert, María B., «Políticas de empleo (I)», 265.

10 Ramos Martín, Francisco, «Políticas de empleo», pp. 356 - 357.

11 Ibíd., p. 358. 
circunstancias especiales (emigrantes retornados, excarcelados, inválidos parciales, etc.).

Mientras que el nivel contributivo está basado claramente en la noción de seguro, ya que pretende garantizar al desempleado una renta sustitutiva del salario dejado de percibir, el nivel asistencial tiene, en el caso del desempleo, un carácter complementario al de la prestación contributiva, ya que tiende a cubrir las deficiencias de cobertura de la prestación contributiva ${ }^{12}$.

$\mathrm{Si}$ bien las prestaciones de desempleo, igual que otras de Seguridad Social, suponen un paso adelante en el proceso de aparición y legitimación de los derechos de ciudadanía, por los cuales a sus beneficiarios se les deben asegurar unas mínimas condiciones de vida, por vía, en este caso, de la entrega temporal de un valor mientras los perceptores no son capaces de conseguir un sustento económico, estas medidas todavía están sometidas y totalmente condicionadas por la ética del trabajo ${ }^{13}$.

Efectivamente, cuando se plantean este tipo de políticas pasivas, las características de las prestaciones de desempleo, que tienen que ver con el tipo de desempleo que el sistema cubre, el perfil de los beneficiarios, la protección sólo de la pérdida de empleo previo y no imputable al trabajador, la configuración contributiva de la prestación, la tasa de sustitución de la prestación, es decir, la relación entre salario y monto de la prestación, su duración; están

12 Ibíd., p. 359.

13 López GANDíA, Juan, «Las políticas pasivas: protección por desempleo», en Políticas sociolaborales, Tratados y manuales (Navarra: Thomson Reuters, 2014), p. 465. 
anclados totalmente en la concepción de la sociedad del trabajo y el deber de trabajar ${ }^{14}$.

En el caso ecuatoriano, esto se puede verificar con claridad, primero en la disposición constitucional contenida en el art. 33, que señala que el trabajo es: "un derecho y un deber social, y un derecho económico, fuente de realización personal y base de la economía"15, es decir que para el Estado ecuatoriano, el trabajo es una obligación que se impone a quienes tienen las condiciones para hacerlo, y considera a esta actividad como el requisito previo para alcanzar la plena ciudadanía y lograr la satisfacción personal que entre otras cosas implica la posibilidad de cubrir las necesidades propias y de la familia.

Ya de forma específica, el artículo 6 de la Ley Orgánica para la Promoción del Trabajo Juvenil, regulación excepcional de la jornada de trabajo, cesantía y seguro de desempleo16, que reformó algunos cuerpos normativos, entre ellos la Ley General de Seguridad Social, incorporó el seguro de desempleo a partir del art. 275 de la Ley, definiéndolo como una "prestación económica que protege a los afiliados del Instituto Ecuatoriano de Seguridad Social, bajo relación de dependencia por la pérdida de ingresos generada por un cese temporal de actividades productivas por causas ajenas a su voluntad..." Es decir que se condiciona la percepción del seguro a quienes, habiendo mantenido previamente relación laboral, han sido cesados involuntariamente de su trabajo.

\footnotetext{
14 Ibíd., pp. 465 - 466.

15 Art. 33 de la Constitución Política de la República del Ecuador.

16 RO-S 720, de 28 de marzo de 2016.
} 
Sin ánimo de agotar el tema que ahora se aborda, tanto por la amplitud del mismo, como porque no es materia del presente estudio, y únicamente para demostrar los efectos limitados de la política pasiva de empleo que constituye el seguro de desempleo en Ecuador, me refiero al artículo innumerado de la Ley reformatoria al Código del Trabajo, que trae los requisitos para la percepción de este beneficio, consistentes en haber realizado veinte y cuatro aportaciones previas, acumuladas y no simultáneas, de las cuales seis deben haber sido hechas inmediatamente antes de la contingencia, el encontrarse el solicitante en situación de desempleo por un período no menor a sesenta y un días y no mayor a setenta y cinco días, y no ser jubilado.

Los requisitos mencionados, claramente atan la percepción del seguro de desempleo a una condicionalidad que es el empleo previo, con cierta estabilidad, y dentro de la formalidad que implica la afiliación a la Seguridad Social, y que termina de forma involuntaria para el trabajador. Entonces, se deja fuera del beneficio a quienes no alcanzan el tiempo mínimo de cotización, o a los que terminan la relación voluntariamente. A estos últimos, sin embargo, les quedaría la posibilidad de solicitar el fondo de cesantía ${ }^{17}$.

17 La cesantía es el fondo que se acumula en la cuenta individual del afiliado. Del 20,60\% del salario de aportación del afiliado del Seguro General, hasta el 27 de marzo de 2016 se destinaba el 3\%, y desde el 28 de marzo en adelante es el $2 \%$ el que permanece en la cuenta individual y el $1 \%$ tiene carácter solidario y sirve para incrementar la cuenta general para pago de desempleo.

El valor acumulado de cesantía se entrega al afiliado, después de dos meses que dejó de aportar al IESS (renuncia, despido, jubilación, etc.) y siempre que tenga acumulado por lo menos 24 aportaciones mensuales. Cuando el afiliado fallece se entrega la cesantía a sus deudos con derecho. 
Ahora bien, la aparición de las prestaciones por desempleo en el marco más global de la política de empleo, supone ir más allá de la estricta prestación económica, que aunque en sí misma tiene un valor de seguridad económica, de protección y de solidaridad, no debe ser ajena a otros objetivos, como son que el beneficiario retorne al mercado de trabajo, no solo subordinado sino también mediante auto emprendimientos, a través de mecanismos que vayan aparejados a la entrega periódica de la prestación y que busquen mejorar la empleabilidad del trabajador desempleado, destinados a ampliar su capacidad profesional o hacer frente a su obsolescencia laboral, tales como estrategias de formación y orientación, reciclaje, técnicas de búsqueda de empleo, tutorías personalizadas, iniciativas de inserción profesional, entre otras $^{18}$.

Es decir, se hace necesario combinar políticas de empleo, que compaginen programas de prestación por desempleo con medidas activas simultáneamente. En ese marco se sitúan las obligaciones del perceptor de participar en las citadas acciones, así como de no rechazar ofertas de empleo convenientes o adecuadas ${ }^{19}$.

Hay convenios específicos sobre prestaciones de desempleo, como el Convenio sobre el desempleo de la OIT, 1934 (núm. 44) ${ }^{20}$, que no ha sido ratificado por Ecuador. Otro es el Convenio sobre la seguridad social (norma mínima), 1952 (núm. 102)21 , que Ecuador

18 López GANDíA, Juan, «Las políticas pasivas: protección por desempleo», p. 466.

19 Ibíd.

20 Convenio por el que se garantizan indemnizaciones o subsidios a los desempleados involuntarios, adoptado en Ginebra, en la $18^{\text {a }}$ reunión CIT (23 junio 1934).

21 Convenio relativo a la norma mínima de la seguridad social, adoptado en Ginebra, en la 35ª reunión CIT (28 junio 1952). 
ha ratificado pero no en la parte IV, relativa a las prestaciones de desempleo. Finalmente está el Convenio sobre el fomento del empleo y la protección contra el desempleo, 1988 (núm. 168) 22, que Ecuador tampoco ha ratificado.

Es importante que, una vez que Ecuador prevé la prestación por desempleo, mejore el alcance de este beneficio, y lo utilice para lograr otros objetivos que no sean exclusivamente los de asegurar una renta mínima al beneficiario por cinco meses, sino que constituya un verdadero apoyo al desempleado para que aproveche el tiempo de paro para mejorar sus habilidades y destrezas, a efectos de volver a reinsertarse en el mercado laboral de forma satisfactoria.

\section{POLÍTICAS ACTIVAS DE EMPLEO}

Las políticas activas de empleo tienen un origen posterior a las políticas pasivas, y están asociadas a los modelos nórdicos de estado de bienestar. Sin embargo, en los últimos tiempos su uso como instrumento de lucha contra el desempleo se ha generalizado. Así, son el conjunto de programas que permiten la consecución del empleo, la adecuación de la oferta y la demanda y la reducción de las situaciones de desempleo ${ }^{23}$.

Las políticas activas de empleo se identifican con el conjunto de medidas y acciones, adoptadas por los poderes públicos, destinadas a favorecer la adaptación de la mano de obra

22 Convenio sobre el fomento del empleo y la protección contra el desempleo, adoptado en Ginebra, en la $75^{\mathrm{a}}$ reunión CIT (21 junio 1988).

23 Ramos Martín, Francisco, «Políticas de empleo», 372. 
(formación profesional); fomentar la creación de empleo; mantener los empleos existentes y conseguir el ajuste simultáneo entre la oferta y la demanda de empleo, a través de una mejor circulación de la información y de unos servicios de empleo más eficaces ${ }^{24}$.

Las políticas activas tienen cinco objetivos principales ${ }^{25}$ :

- Incrementar la transparencia del mercado de trabajo.

- Desarrollar la estabilidad en el empleo.

- Optimizar los recursos humanos.

- Impulsar la creación de empleo y el reparto del trabajo.

- Atender a colectivos desfavorecidos, mejorando su capacidad para insertarse en el mercado de trabajo.

La variada tipología de medidas que existe en el ámbito de las políticas activas suele clasificarse en tres grandes grupos ${ }^{26}$ :

- Políticas de orientación, intermediación y colocación. Actuaciones que tratan de mejorar la relación entre oferta y demanda de trabajo. Por ejemplo, orientación en la búsqueda de empleo, gestión de ofertas y contratación, etc.

- Políticas de formación. Actuaciones de formación profesional ocupacional o continua, dirigidas a proveer de los conocimientos, capacidades y habilidades que permitan a aquellos en situación de desempleo (o en riesgo de ser desempleados) mejorar sus oportunidades de inserción en el mercado laboral.

- Políticas de promoción, creación y mantenimiento

24 Cabeza Pereiro, Jaime y Cardona Rubert, María B., «Políticas de empleo (I)», 265.

25 Ruesga Benito, Santos y otros, Economía del trabajo y política laboral, $2^{\mathrm{a}}$. edición, Economía y Empresa (Pirámide, 2014), 188-189.

26 Ramos Martín, Francisco, «Políticas de empleo», 373. 
de empleo. Actuaciones que persiguen crear empleo mediante incentivos a la contratación, creación de empleo público directo, fomento del autoempleo, o ajuste de las condiciones de trabajo para evitar despidos.

\section{POLÍTICAS DE ORIENTACIÓN, INTERMEDIACIÓN Y COLOCACIÓN.}

El primer grupo de medidas están destinadas a corregir las disfuncionalidades del mercado laboral provocadas por la falta de información, por medio de la facilitación de contacto entre la oferta y la demanda de empleo, a través de servicios especializados, que pueden ser públicos, privados o mixtos ${ }^{27}$.

Este servicio responde a una necesidad objetiva y estructural de los mercados de trabajo, que es la de disponer de canales de información entre oferta y demanda de mano de obra; y en ese sentido, satisface requerimientos individuales de los actores de este mercado, demandantes de empleo y empleadores potenciales, para los que este servicio es de gran importancia, lo que le da un claro valor económico a este servicio, pudiendo ser prestado por entidades privadas con ánimo de $\operatorname{lucro}^{28}$.

La intermediación, como se conoce al servicio de colocación, habiendo surgido como una actividad en el mercado de trabajo,

27 Cabeza Pereiro, Jaime y Cardona Rubert, María B., «Políticas de empleo (I)», pp. 269-270.

28 CAmas Roda, Ferrán y RodríGueZ-PIÑERo Royo, Miguel, «Políticas de empleo (II): políticas activas», en Políticas Sociolaborales, Tratados y manuales (Navarra: Thomson Reuters, 2014), p. 294. 
posteriormente se convierte en un servicio público ${ }^{29}$, llegando a adquirir en la actualidad el carácter de un derecho para los ciudadanos ${ }^{30}$.

En opinión de una parte de la doctrina,el derecho a la intermediación sería una manifestación de uno más amplio que es el derecho a la asistencia en la búsqueda de empleo, y que responde a mercados de trabajo cada vez más temporales, en donde los beneficiarios principales son los demandantes de empleo ${ }^{31}$.

En Ecuador, la colocación es vista no como un derecho, sino como un servicio público. Así lo prevé el artículo 557 del CT, que precisamente determina su carácter público y gratuito, no obstante que no prohíbe la prestación del servicio de colocación por entidades privadas $^{32}$.

A partir de la fusión del antiguo Ministerio del Trabajo y la Secretaría Nacional Técnica de Desarrollo de Recursos Humanos y Remuneraciones del Sector Público ${ }^{33}$, el servicio público de empleo adquirió nuevas características, ya que pasó de una estructura de oficinas de empleo ubicadas en las capitales de provincias, a un sistema más integrado de empleo que lleva por nombre Red Socio Empleo, creado en 2010 y que cuenta con una estructura descentralizada de centros de empleo, además de otros

29 Esta concepción está presente en el Convenio No 88 Sobre el Servicio de Empleo (1948) de la OIT, ratificado por Ecuador en 1975.

30 Ibid., p. 295.

31 Ibid., p. 296.

32 Art. 558 del CT.

33 Mediante Decreto Ejecutivo 10, publicado en el RO 10, de 24 de agosto de 2009 . 
canales para acceder a los servicios de empleo, incluido un portal de empleo en línea y un número de teléfono gratuito, que dan información sobre empleo y facilitan el registro de personas que buscan empleo y vacantes en el sector público y privado ${ }^{34}$.

Los pasos dados en los últimos años para mejorar el servicio nacional de colocaciones es importante, ya que como se ha visto, es un mecanismo que permite superar las desigualdades provocadas por la falta de información entre oferentes y demandantes de trabajo; no obstante, es necesario lograr una colocación más dinámica, para lo que hay que conocer mejor las necesidades de los potenciales demandantes del servicio. Para ello, hay que invertir en personal calificado y mejor capacitado para atender a empleadores y buscadores de empleo, superando la información mecánica hasta proveer una cualificada intermediación entre quienes buscan empleo y los que lo ofertan.

Para avanzar hacia un servicio de mejor calidad, la Red debe mejorar la capacidad para proporcionar servicios en persona a un número cada vez mayor de buscadores de empleo que necesitan un apoyo más directo35, para ello, incluso con el apoyo de la tecnología, deberían realizarse entrevistas de trabajo que permitan extraer la mayor información del candidato para presentar posteriormente todas sus capacidades y habilidades a potenciales empleadores.

34 «Servicios públicos de empleo en América Latina y el Caribe. Ecuador, características principales y desafíos» (Organización Internacional del Trabajo, 2015), 2-3.

Ibíd., 9. 
Lo deseable, sin embargo, es avanzar hacia la consideración de la colocación laboral como un derecho, al que deberían acceder todas las personas en aptitud de trabajar, que sea disponible y de fácil acceso, que forme parte de un sistema integrado de formación-capacitación-colocación.

\section{POLÍTICAS DE FORMACIÓN}

La formación profesional entendida de forma general, como la enseñanza que permite a quien la recibe desempeñarse de forma cualificada de acuerdo a las necesidades del mercado de trabajo, incluye tanto la realizada en el sistema educativo, que es la llamada formación profesional inicial o formación profesional reglada; como la formación profesional en el ámbito laboral, o llamada formación profesional para el empleo ${ }^{36}$.

La formación profesional para el empleo es el conjunto de acciones que buscan impulsar y extender entre las empresas y los trabajadores ocupados y desempleados una formación que responda a sus necesidades y contribuya al desarrollo de una economía basada en el conocimiento, lo que implica el acceso a toda la formación que pueda ser requerida por los trabajadores para lograr su realización personal e inclusión social, o destinada a satisfacer las necesidades del empleo ${ }^{37}$.

En Ecuador, el derecho a la formación profesional se integra en el reconocimiento constitucional más amplio al derecho a la

36 Camas Roda, Ferrán y RodrígueZ-PIÑERo Royo, Miguel, «Políticas de empleo (II): políticas activas», p. 318.

37 Ibíd., p. 321. 
educación (art. 26 CE), del que es una manifestación cualificada y se relaciona con la obligación que la Carta Magna impone a los poderes públicos de fomentar una política que garantice la igualdad e inclusión social por medio de la educación, reconociendo también la Constitución como formas de aprendizaje la educación escolarizada y no escolarizada ${ }^{38}$.

El art. 329 inciso quinto de la CE exige al Estado el impulso a la formación y capacitación para mejorar el acceso y calidad del empleo y las iniciativas de trabajo autónomo.

Por su parte, el Plan Nacional del Buen Vivir 2013 - 2017, al desarrollar el Objetivo 9: garantizar el trabajo digno en todas sus formas, plantea la necesidad de generar empleo en condiciones dignas, buscar el pleno empleo, priorizando a los históricamente desfavorecidos, reducir el trabajo informal y garantizar el cumplimiento de los derechos laborales. Para ello, pretende lograr el fortalecimiento de los esquemas de formación ocupacional y capacitación por medio de la articulación con las necesidades del sistema de trabajo y en relación con la productividad laboral ${ }^{39}$.

En cambio, el art. 4 de la Ley de Educación Superior40 prevé que el derecho a la educación superior consiste en el ejercicio efectivo de la igualdad de oportunidades, en función de los méritos respectivos, a fin de acceder a una formación académica y profesional con producción de conocimiento pertinente y de excelencia.

\footnotetext{
38 Art. 28, inciso tercero de la CE.

39 Plan Nacional de Desarrollo 2013 - 2017, versión reducida, Secretaría Nacional de Planificación y Desarrollo - SEMPLADES, Quito (2009), 75.

RO-S 298, de 12 de octubre de 2010.
} 
Es decir, que el Estado ecuatoriano reconoce la relación que existe entre el proceso de formación y la posibilidad de inserción en el mercado laboral en mejores condiciones. Más adelante se abordará con mayor amplitud lo relativo a esta relación, cuando se analice las políticas de empleo para jóvenes.

Por ahora, hay que mencionar que entre otros esfuerzos, Ecuador ha apostado desde $1978^{41}$ por generar procesos de capacitación y formación profesional a nivel nacional dirigido a buscadores de empleo que no han podido iniciar o continuar estudios dentro del sistema formal de educación, a través del Servicio Ecuatoriano de Capacitación Profesional (SECAP), que es otro socio de la Red Socio Empleo, cuenta con centros y unidades móviles de formación con capacidad para atender una media de 2000 personas al año. Se organizan cursos de formación en sectores con mucha demanda de trabajadores con calificaciones medias, como el comercio, la industria, tareas administrativas, los servicios y la construcción. Los graduados del SECAP se derivan a la Red Socio Empleo para que reciban apoyo en la búsqueda de empleo ${ }^{42}$.

En el año 2009 se creó el Sistema Nacional de Formación Profesional $^{43}$, con el objetivo de formar el talento humano del país, incrementar la pertinencia de la oferta, y promover mejoras continuas en términos de producción, productividad y empleabilidad ${ }^{44}$.

41 DS 2928 publicado en el RO 694 de 19 de octubre de 1978.

42 «Servicios públicos de empleo en América Latina y el Caribe. Ecuador, características principales y desafíos», 3 .

43 DE 1509, publicado en el RO503 de 9 de enero de 2009.

44 JARA IÑIGUEZ, Irma, «Cómo deben ser consideradas las características 
La hipótesis de la cual partió el trabajo del Sistema Nacional de Formación Profesional, fue:

la escasa orientación de la capacitación al trabajo como un obstáculo para la generación de productividad y competitividad en el trabajador, lo que se refleja tanto en la calidad de la capacitación laboral y pertinencia de estos servicios, como en la cobertura de los trabajadores atendidos. Por ello, desde la agenda pública se determinó la capacitación laboral como elemento importante para a la reducción de la pobreza, considerando indispensable una nueva política de formación del talento humano cuyo objetivo-meta sea el mejorar la calidad y pertinencia de estos servicios, aumentar paulatinamente la cobertura e incrementar el acceso a los mismos ${ }^{45}$.

Sin duda, hay una evolución significativa en materia de política pública de capacitación laboral que se ha reflejado en el ejercicio de este Ejecutivo; no obstante, una vez institucionalizada la misma, hay que mejorar la oferta de capacitación. Efectivamente, en sintonía con las iniciativas internacionales orientadas a la formación técnica o capacitación laboral, es necesario enfatizar el desarrollo de habilidades suaves y habilidades para la vida. Estas habilidades consisten en trabajar en niveles aptitudes como "liderazgo, creatividad,

y limitaciones locales en las teorías de construcción de las políticas públicas. Estudio de las políticas de capacitación en el Ecuador: 1978-2011» (Universidad Andina Simón Bolívar, Sede Ecuador, 2015), 118.

Ibíd., p. 177. 
perseverancia, capacidad de enfrentar y resolver problemas nuevos o tomar decisiones, etc."46

Para ello, se deben mejorar y afinar los niveles de participación en todas las fases la política pública tendente a mejorar la empleabilidad por vía de la capacitación, de los principales actores que son trabajadores, demandantes de trabajo y empleadores, en donde consten representantes de distintos grupos de edad, género, situación educativa y socioeconómica, porque son los más legitimados para opinar respecto de su realidad y necesidades. Como se ha constatado, las medidas que se toman de manera vertical y sin contar con los principales afectados y beneficiarios no suelen tener el efecto deseado.

\section{POLÍTICAS DE PROMOCIÓN, CREACIÓN Y MANTENIMIENTO DE EMPLEO}

Dentro de las políticas de promoción, creación y mantenimiento de empleo, hay una subclasificación. Están las medidas que favorecen la contratación de trabajadores o la creación de empleo por vía de medidas de fomento; y por otro lado están las políticas de flexibilización.

Las primeras medidas tienen como objetivo facilitar e incrementar la contratación laboral de personas desempleadas. Estas políticas tratan de actuar sobre la oferta de empleo y ocupación. No son

46 Organización Internacional del Trabajo, ¿Qué sabemos sobre los programas y políticas de Primer Empleo en América Latina?, Juventud e informalidad (Perú: Oficina Regional para América Latina y el Caribe, 2015), 14. 
neutrales, sino que pretenden alterar el funcionamiento "natural" del mercado de trabajo en una determinada dirección. Para lograr su cometido, se puede acudir a instrumentos muy variados, como ayudas económicas, prestación de servicios diversos, reconocimiento de un status jurídico diferenciado, entre otros ${ }^{47}$.

Estas políticas pueden incentivar la contratación, creación de empleo o mantenimiento de puestos de trabajo, ya sea de forma general, o dirigidas a sectores o colectivos específicos; no obstante, la práctica totalidad de las medias de fomento se enfocan en determinados colectivos con especiales dificultades para acceder al empleo, como jóvenes, mujeres, discapacitados y personas de otras culturas.

Ecuador ha aplicado medida de fomento destinada a la contratación de personas con discapacidad, prevista primero en la Ley 2006-28 reformatoria del Código del Trabajo ${ }^{48}$, y posteriormente en la Ley Orgánica de Discapacidades (LOD), vigente desde septiembre de $2012^{49}$, que trae un capítulo sobre el trabajo de este colectivo, con especial énfasis en la política pública dirigida a facilitar su inclusión laboral. La disposición sobre contratación de personas con discapacidad exige contratar por lo menos un cuatro por ciento (4\%) del total de la plantilla, de personas con discapacidad, para realizar actividades permanentes y apropiadas en relación con los conocimientos, condiciones físicas y aptitudes individuales de las personas que contraten dentro de este porcentaje $\mathrm{e}^{50}$.

47 Camas Roda, Ferrán y Rodríguez-PIÑERo Royo, Miguel, «Políticas de empleo (II): políticas activas», 311-312.

RO 198, de 30 de enero de 2006.

RO-S 796, de 25 de septiembre de 2012.

50

La única excepción que se aplica al porcentaje sobre el total de la 
Otro grupo de políticas son las de flexibilidad en las condiciones de trabajo, que son mecanismos de respuesta a la coyuntura que tienden a alterar las condiciones laborales previamente establecidas, generalmente en detrimento de los derechos de los trabajadores. Estas medidas son las más polémicas porque en base a argumentos de superación de situaciones coyunturales, que en realidad son circunstancias estructurales, sirven para aplicar medidas flexibilizadoras de carácter general ${ }^{51}$.

Efectivamente, el empleador estará siempre interesado en obtener un máximo de flexibilidad en la gestión del talento humano, pues es un instrumento muy útil para optimizar recursos; mientras que el trabajador, que depende de su salario como medio de subsistencia, está siempre interesado en un máximo de continuidad y de seguridad jurídica en su relación laboral. Por tanto, la flexibilidad es algo que interesa a los empresarios y no a los trabajadores. En ese sentido, el Estado cumple un papel fundamental al encontrar un punto de equilibrio entre los intereses contrapuestos de las partes ${ }^{52}$, y sobre todo, debe contar con suficiente información para no tomar decisiones que aparentemente pueden mejorar la situación de empleo a nivel general, pero cuyos efectos son más perniciosos que positivos por precarizar la situación de los trabajadores.

nómina, se refiere a las Fuerzas Armadas, Policía Nacional, Cuerpos de Bomberos y Policías Municipales del sector público, empresas de seguridad y vigilancia privada, donde se aplica el porcentaje únicamente sobre el personal administrativo, excluyendo el desempeño de funciones operativas en razón del riesgo que implica para integridad física de la personas con discapacidad.

51 Chacartegui Jávega, Consuelo y Valle Muñoz, Francisco, «Políticas de flexibilidad», en Politicas Sociolaborales. Un enfoque multidisciplinar, Economía y Empresa, No. 17 (Barcelona: UOC, 2004), 478.

Ibíd. 
La reducción y ampliación de la jornada laboral encajan en este tipo de medidas. Así, la Ley de Orgánica para la promoción del trabajo juvenil, regulación excepcional de la jornada de trabajo, cesantía y seguro de desempleo ${ }^{53}$, que vino a reformar varios cuerpos normativos, incorporó un artículo a continuación del 47, que prevé que en casos excepcionales, y por un período no mayor a seis meses renovables por seis meses más por una sola ocasión, la jornada de trabajo ordinaria común (de ocho horas diarias y cuarenta a la semana) puede ser disminuida, hasta un límite no menor a treinta horas semanales, con la consiguiente disminución de la remuneración, aunque no así de los aportes a la seguridad social y pago de indemnizaciones en caso de despido.

Esta medida, claramente perjudica la situación económica del trabajador, por vía de reducir su remuneración junto con la jornada laboral. Si se piensa en que buena parte de los trabajadores perciben la remuneración básica, o en general salarios bajos, la reducción aun mayor de éstos claramente pone en serias dificultades la satisfacción de las necesidades básicas de los trabajadores y sus familias. Y ante la amenaza de quedarse sin empleo, seguramente se verán obligados a aceptar esta medida, que aunque temporal, puede poner en serios aprietos el cumplimiento de sus proyectos de vida.

Otra medida, prevista a continuación de la anterior, admite que se pacte de manera excepcional y por la naturaleza del trabajo, que las jornadas excedan las ocho horas diarias, siempre que no superen el máximo de cuarenta semanales ni de diez al día, en horarios que se pueden distribuir de manera irregular en los cinco días laborables de la semana.

RO-S 720, de 28 de marzo de 2016. 
Esta última medida pretende cubrir las actuales necesidades de los sectores productivos y de comercio, así como la prestación de servicios que cada vez más requieren una atención permanente, sin que eso implique mayor incremento en la remuneración de los trabajadores. Ahora bien, como acertadamente lo recoge la doctrina nacional, no se ha reparado que para ello ya existe en la legislación nacional suficientes figuras laborales que permiten atender tales requerimientos, como:

"el reconocimiento a los trabajadores de recargos por jornadas suplementarias y extraordinarias; la posibilidad de establecer turnos; el régimen de contratación parcial, o sencillamente la gran variedad de modalidades contractuales; ello sin mencionar la posibilidad de establecer horarios especiales de trabajo...; es decir que resulta por demás innecesaria, además de atentatoria a los derechos ya adquiridos de los trabajadores, semejante pretensión" 54 .

Así es, si en otros entornos se evalúa la posibilidad de reducir la jornada laboral, sin afectación a la remuneración, o de cambiar la lógica de recompensa del trabajo, que no mida primordialmente el tiempo de dedicación del trabajador, parece una involución que en Ecuador se planteen medidas como la reducción impuesta de jornada y remuneración; o la de prolongar la jornada laboral diaria con el único ánimo de abaratar al empleador el costo del trabajo prolongado más allá de los límites legales, sociales y psicológicos admitidos.

54 Monesterolo, Graciela, "Jornadas laborales prolongadas y sus repercusiones en el buen vivir del trabajador y de su familia», Revista de Derecho FORO 19 (I semestre de 2013), pp. 67-68. 


\section{POLÍTICAS DE EMPLEO DESTINADAS A JÓVENES}

La crisis de empleo que golpea a los jóvenes en todo el mundo es una de las principales preocupaciones de los organismos internacionales especializados. De ahí que el empleo juvenil es una de las prioridades, en especial porque éste sea de calidad.

En términos generales, los jóvenes entre 15 y 24 años se encuentran en situación de desventaja frente a los mayores de 25, entre otras causas, porque tienen menos o ninguna experiencia laboral, y a menudo cuentan con menores niveles de educación. Dentro de ese grupo etario, los jóvenes que abandonan el sistema educativo se enfrentan a dificultades particulares para acceder al mercado de trabajo.

Efectivamente, pese a que los jóvenes tienen hoy niveles más altos de educación formal que las anteriores generaciones, enfrentan, mayores dificultades de acceso al empleo. Esto se explica porque los sistemas de educación y formación para el trabajo no han sido capaces de acoplarse a los cambios productivos y socioculturales recientes, de manera que no facilitan la transición al mundo laboral ${ }^{55}$.

La mayoría de experiencias de trabajo iniciales no cumplen con las expectativas esperadas, ya que son muchos los jóvenes que reciben salarios bajos, poca acumulación de conocimientos y habilidades, amenazas de despido, malos tratos, acoso laboral

55 Weller, Jürgen, «La inserción laboral de los jóvenes: tensiones y perspectivas», en Mundos del trabajo y políticas públicas en América Latina (Quito: FLACSO Ecuador y Ministerio de Cultura, 2008), 36 y 37. 
y/o sexual, estas condiciones no permiten aprovechar todo el potencial que la actividad productiva podría brindar para el desarrollo individual y social de los jóvenes ${ }^{56}$.

La poca atracción que estas primeras experiencias laborales generan en los jóvenes ha marcado una realidad, conocida como la de los "ninis" ni estudian ni trabajan, lo que retrasa o pervierte esa entrada al mundo productivo.

Describir la situación de los jóvenes en el mercado de trabajo es relativamente fácil: es difícil, compleja, llena de incertidumbres, y más si se trata de mujeres.

Hay ciertos elementos que caracterizan esta situación ${ }^{57}$ :

- Las tasas de desempleo de los jóvenes suelen ser casi el triple de las de los adultos.

- La inserción laboral de los jóvenes es precaria, siendo las tasas de empleo informal o de empleos sin acceso a seguridad social, considerablemente mayores a las de los adultos.

- La rotación laboral y la duración del desempleo son mayores entre los jóvenes que entre los adultos.

- Una proporción no despreciable de jóvenes ni estudia ni trabaja, ni busca trabajo y en algunos casos, ni desea estudiar.

$56 \quad$ Ibíd., 37.

57 Organización Internacional del Trabajo, ¿Qué sabemos sobre los programas y políticas de Primer Empleo en América Latina?, Juventud e informalidad (Perú: Oficina Regional para América Latina y el Caribe, 2015), 2. 
En el caso ecuatoriano, los jóvenes entre 15 y 24 años de edad también tienen mayores tasas de subocupación ${ }^{58}$ y desocupación ${ }^{59}$ que los mayores de esa edad, y como se verá más detalladamente a continuación, en el caso de las mujeres jóvenes incluso se ha incrementado en este año. Así, a diciembre de 2007 el porcentaje de subocupados entre hombres jóvenes era de $53 \%$ y a junio de 2016 bajó a 45,1\%, mientras que el de desocupados entre 15 y 24 años fue de $9,9 \%$ al inicio del período que se analiza y de 7,8 $\%$ al final. En el caso de las mujeres jóvenes, el porcentaje de subocupadas a diciembre de 2007 fue de $54,1 \%$ y en junio de 2016 de $51 \%$; en cambio las jóvenes en desocupación pasaron del 14,3 $\%$ al $17,2 \% .^{60}$

Al cotejar estos porcentajes con los de adultos mayores de 25 años, encontramos que en el caso de los hombres, a diciembre de 2007 un $37.1 \%$ estaban subocupados y un 2,4\% desocupado, mientras que a junio de $201629,4 \%$ son subocupados y $3,5 \%$ son desocupados; habiéndose incrementado la tasa de desocupados.

58 Personas con empleo que, durante la semana de referencia, percibieron ingresos inferiores al salario mínimo y/o trabajaron menos de la jornada legal y tienen el deseo y disponibilidad de trabajar horas adicionales. Es la sumatoria del subempleo por insuficiencia de tiempo de trabajo y por insuficiencia de ingresos.

59 Personas de 15 años y más que, en el período de referencia, no estuvieron Empleados y presentan ciertas características: i) No tuvieron empleo, no estuvieron empleados la semana pasada y están disponibles para trabajar; ii) buscaron trabajo o realizaron gestiones concretas para conseguir empleo o para establecer algún negocio en las cuatro semanas anteriores. Se distinguen dos tipos de desempleo: abierto y oculto

60 Encuesta Nacional de Empleo, Subempleo y Desempleo (ENEMDU) diciembre 2007 a junio 2016, elaborado por Unidad de Información Socio-Ambiental UISA, Universidad Andina Simón Bolívar. 
En el caso de las mujeres, a diciembre de 2007 el 50,5\% estaba subocupada y el 6,3\% desocupada, y para junio de 2016 el 41,1\% está subocupada y el $6 \%$ está desocupada. ${ }^{61}$

De los datos aportados, se puede ver que el desempleo y subempleo golpean con fuerza a los menores de 25 años, y de manera especial a las mujeres jóvenes. En el caso de hombres jóvenes, la tasa de desempleados casi cuadriplicaba el de hombres mayores de 24 años en diciembre de 2007; mientras que la tasa de mujeres jóvenes desempleadas era en esa época de más de doble. A junio de este año, en cambio, el porcentaje de desempleados mejores de 24 años superó el doble del de los adultos, y en el caso de las mujeres, entre las jóvenes el desempleo es de casi el triple que las adultas.

Estos datos aportan elementos importantes que deben ser tomados en cuenta por los hacedores de políticas públicas, a la hora de diseñar y planificar medidas de fomento del empleo, ya que es imprescindible que además de la edad, se desagregue las cifras por género.

Frente a la realidad del desempleo juvenil a nivel mundial, y con origen en la Resolución de 2005 relativa al empleo de los jóvenes y en sus conclusiones ${ }^{62}$, el actual Programa de Empleo Juvenil de la OIT proporciona asistencia a todos los países en el desarrollo de intervenciones coherentes y coordinadas sobre el empleo de

61 Ibíd.

62 Resolución relativa al "empleo de los jóvenes" y conclusiones sobre la "promoción de vías para acceder a un trabajo decente para los jóvenes", Conferencia Internacional del Trabajo OIT, 93 $93^{\mathrm{a}}$ reunión, 15 de junio de 2005. 
este colectivo. Pese a la vigencia de la Resolución de 2005, en el año 2012, se adoptó otra relativa a la crisis del empleo juvenil, también seguida de sus respectivas conclusiones ${ }^{63}$.

En lo que tiene que ver con las políticas macroeconómicas, la Resolución de 2012 incide en su importancia para favorecer el empleo, en especial, en la industria y en sectores sensibles de la economía, imprescindibles para cualquier transformación estructural, sin olvidar la necesidad de cierta inversión pública en infraestructuras y programas de empleo. Entre los aspectos que inciden en la empleabilidad de los jóvenes, menciona su educación, formación, competencias y la transición de la escuela al trabajo.

Las medidas relacionadas con la edad adquieren especial interés en su relación con el concepto de empleabilidad, entendida esta como el conjunto de aptitudes y capacidades que fortalecen la posición de los individuos en el acceso al mercado de trabajo, supone una proyección de los objetivos de la política de empleo hacia un ámbito subjetivo concreto, proporcionando instrumentos adecuados en función de los caracteres que configuran el colectivo protegido y tratando de incrementar sus posibilidades de ocupación ${ }^{64}$.

63 Resolución y conclusiones "la crisis de empleo juvenil: un llamado a la acción", Conferencia Internacional del Trabajo OIT, $101^{a}$ reunión, 14 de junio de 2012.

64 PRADOS DE REYES, Francisco, «Modalidades contractuales y política de empleo juvenil», en Retos del Derecho del Trabajo frente al desempleo juvenil, Monografías de temas laborales 52 (Granada: Consejo Andaluz de Relaciones Laborales, 2014), 607. 
En ese ámbito, la edad actúa como elemento determinante para la caracterización de figuras contractuales o de sistemas de contratación estrechamente vinculados a circunstancias de especial incidencia en los sectores de población joven y de manifiesto protagonismo entre las que motivan sus dificultades de acceso al empleo ${ }^{65}$.

La capacitación para el trabajo y la adquisición de la experiencia profesional son de las más relevantes actuaciones destinadas a mejorar la productividad del trabajador joven y su propia competitividad, que tienen como proyección común la de centrarse en los primeros estadios de la vida profesional del trabajador; típicamente sobre el colectivo de los trabajadores jóvenes ${ }^{66}$.

A criterio de la OIT, los subsidios salariales y otros incentivos financieros (por ejemplo exenciones fiscales o exenciones de las contribuciones a la seguridad social durante un período limitado) aplicados para que los empleadores contraten a jóvenes, pueden ayudar a mejorar la transición de la escuela al trabajo, en especial durante tiempos de recesión económica. De igual modo, pueden resultar una valiosa herramienta para contrarrestar el costo de la formación inicial de los trabajadores jóvenes o para compensar su limitada experiencia laboral y baja productividad inicial ${ }^{67}$.

\footnotetext{
65 Ibíd.

66 Ibíd., 608.

67 Tendencias mundiales del empleo juvenil 2015 Promover la inversión en empleos decentes para los jóvenes (Ginebra: Oficina Internacional del Trabajo, 2015), 77 - 78.
} 
Efectivamente, si se orientan adecuadamente, los subsidios salariales pueden ser especialmente eficaces para mejorar las tasas de empleo de los trabajadores jóvenes que se enfrentan a desventajas en el mercado de trabajo. No obstante, la mala utilización de los subsidios salariales puede llevar a distorsiones en el mercado, como los efectos de sustitución, cuando se prefiere a un trabajador subvencionado frente a otro que se ajustaría mejor a la vacante ${ }^{68}$.

En el país, aparte de las disposiciones analizadas, la Ley de la Juventud ${ }^{69}$ prevé políticas específicas de promoción del empleo juvenil, dirigidas a crear oportunidades de trabajo que consideren las particularidades de este grupo, facilitar el paso del estudio al trabajo por medio de pasantías, conceder créditos para proyectos productivos, asegurar que el trabajo no interfiera en su educación, salud y recreación, evitar la discriminación en el empleo y mejorar las condiciones laborales a las jóvenes gestantes y a las madres lactantes, respetar y cumplir los derechos laborales y la seguridad social y salud ocupacional. Esta ley mantiene sintonía con la voluntad expresada en la Constitución y otros cuerpos normativos, por promover el ingreso de los jóvenes al mundo productivo en las mejores condiciones posibles.

A continuación se examinará con mayor atención las medidas implementadas en la Ley Orgánica para la Promoción del Trabajo Juvenil, regulación excepcional de la jornada de trabajo, cesantía y seguro de desempleo, que buscan favorecer el acceso a trabajo decente de los jóvenes, sobre todo de aquellos con poca y ninguna experiencia laboral.

68

69

Ibíd.

Ley 49, publicada en el RO 439, de 24 de octubre de 2001. Tiene algunas reformas, la última de 07 de julio de 2014. 


\section{LAS PRÁCTICAS PRE PROFESIONALES}

Hay consenso entre interlocutores sociales, e instituciones internacionales y nacionales en relación con los aspectos positivos que para la inserción laboral de los jóvenes tiene la realización de prácticas pre profesionales. Pero también son conscientes todas las partes implicadas de que los períodos de prácticas a menudo se convierten en una vía de explotación de los becarios como mano de obra barata e incluso se utilizan para sustituir puestos de trabajo reales ${ }^{70}$.

Los elementos que permitirían configurar un marco de calidad para las prácticas pasarían por el respecto de las siguientes garantías:

- "Un período en prácticas debe basarse en un contrato escrito

- Transparencia en la información sobre los derechos y obligaciones de las partes implicadas

- Establecer objetivos bien definidos y un contenido de aprendizaje de calidad

- Asignar un tutor o supervisor a la persona en prácticas en la organización de acogida y evaluar el rendimiento de la persona en prácticas al final del período

- Duración: las prácticas deben tener un período específico, para evitar que sustituyan a empleos regulares

- Limitar períodos de prácticas sucesivas con un mismo

70 SÁNCHEZ-RoDAs, Cristina, «Formas no laborales de inserción de los jóvenes en el marcado de trabajo», en Retos del Derecho del Trabajo frente al desempleo juvenil, Monografías de temas laborales 52 (Granada: Consejo Andaluz de Relaciones Laborales, 2014), 372. 
empleador

- Disposiciones sobre cobertura de Seguridad Social para las personas en prácticas

- Remuneración/compensación de costes: debe estarclaramente especificado en el contrato"71.

Como ha señalado la doctrina española, "La distinción entre las prácticas académicas externas y la relación laboral se apoya en la determinación de cuál es el interés o beneficio principal que se satisface con las mismas, si el de quienes realizan las prácticas o el de la entidad que las acoge" 72

En Ecuador, el primer cuerpo normativo específico en materia de pasantías que se dictó es la Ley de Pasantías en el Sector Empresarial ${ }^{73}$, que se encuentra vigente y que fue parcialmente reformada por la Ley Orgánica para la promoción del trabajo juvenil, regulación excepcional de la jornada de trabajo, cesantía y seguro de desempleo, que básicamente redujo el monto de la compensación económica que la empresa debe entregar al pasante, con el ánimo de favorecer la inclusión de estudiantes universitarios en actividades productivas.

Efectivamente, antes de la reforma, la norma preveía como pensión de pasantías, una cantidad no inferior a la del salario mínimo sectorial, a lo que debían sumarse los demás beneficios

71 Ibíd., 373-374.

72 Moreno Gené, Josep, «La nueva regulación de las Prácticas Académicas Externas de los estudiantes universitarios: la potenciación de la finalidad formativa y de mejora de la empleabilidad», Revista de Trabajo y Seguridad Social No. 350 (2012), 18.

RO 689, de 5 de mayo de 1995. 
previstos para los trabajadores, como décimo tercero y cuarto sueldos, en proporción al horario de labores con relación a la jornada laboral completa, debiendo afiliar además al pasante a la seguridad social. Con la reforma, la pensión fue reducida a un tercio de la remuneración básica mensual, manteniéndose no obstante la obligación de afiliar al pasante sobre el salario básico unificado vigente.

Esta situación de precariedad, por mandato de la ley, no puede durar más de seis meses, debiendo ser regulado todo el proceso por organismo regulador del Sistema de Educación Superior.

Finalmente, la reforma abre la posibilidad de que el Ministerio del Trabajo determine porcentajes mínimos de inclusión de pasantes en cada empresa de acuerdo al tipo de actividad y al tamaño de las mismas.

Paralelamente, en el segundo semestre del 2007, el Ministerio del Trabajo implementó el programa "Mi primer Empleo", financiado por el Gobierno Central, con el ánimo de facilitar la transición de los graduados al trabajo. Este programa se dirige a personas entre 18 y 29 años que se encuentran en los últimos años de estudios en alguna institución de educación superior y que no cuentan con experiencia en el sector público, con preferencia de aquellos que hayan obtenido buenas calificaciones durante sus estudios y/o se encuentren en situación de vulnerabilidad; y consiste en la vinculación no laboral de estos estudiantes, en jornadas máximas de cuatro horas al día y veinte a la semana, con un estipendio del 70 por ciento de salario básico unificado y 
la afiliación a la seguridad social ${ }^{74}$.

De las medidas examinadas se puede concluir que éstas enfatizan el paso del mundo escolar al laboral mediante el relajamiento de las condiciones para aceptar pasantes, respecto de los empleadores privados; y por vía de contar con fondos específicos para no cargar a las entidades públicas que admitan a estudiantes en prácticas preprofesionales.

En ambos casos, hay una ausencia de programas específicos que incluyan programas de aprendizaje o certificaciones de aptitudes que los pasantes puedan exhibir una vez cumplido este período, y que les puedan servir para vincularse al mundo laboral. Más bien se apuesta porque sea la misma empresa o institución que admitió al pasante la que lo contrate posteriormente.

Este énfasis en los beneficios de la entidad contratante antes que en los pasantes, puede poner en riesgo los derechos de los estudiantes, y como se apuntó antes, incluso general relaciones laborales encubiertas en contratos de pasantías.

\section{EL PROGRAMA DE SUBSIDIOS DE PRIMER EMPLEO}

Como se mencionó anteriormente, los subsidios salariales y otras ayudas a la contratación de primeros empleos es una medida que utilizan los países para promover el empleo de los jóvenes. Según este esquema, la creación de puestos de trabajo y los procesos de contratación dependen de los empleadores privados, pero los

74 Norma para la implementación de las pasantías del proyecto $\mathrm{Mi}$ Primer Empleo. Acuerdo Ministerial 53, publicado en RO-S 698, de 24 de febrero de 2016. 
costos que esas contrataciones generan son compartidos por los empresarios y el Estado ${ }^{75}$.

Efectivamente, mediante la reducción de costos de contratación, se busca estimular la contratación de colectivos con especiales problemas de desempleo, que en este caso son jóvenes, que también pueden tener bajos niveles de calificación, y por su edad, también son escasos de experiencia ${ }^{76}$.

En nuestro país, la Ley Orgánica para la Promoción del Trabajo Juvenil, trajo consigo la primera política de subsidio de primer empleo aplicable al sector privado para contrataciones laborales. Anteriormente, el Estado ecuatoriano había apostado por cuotas obligatorias a la hora de buscar la contratación de colectivos con dificultades, como es el caso de las personas con discapacidad. En el caso de las mujeres, impide el despido de las trabajadoras gestantes y madres de hijos menores de un año, por medio del despido ineficaz ${ }^{77}$.

Este cambio de estrategia para favorecer la contratación de un colectivo con dificultades de acceso a empleo digno es una apuesta importante que sin duda genera respuestas distintas en los potenciales empleadores, que ya no son constreñidos por temor a la sanción o a la declaración de ineficacia de sus actos, sino que se les propone un subsidio por la contratación de trabajadores jóvenes.

Después de algunos debates, la reforma introducida a partir del

75 Organización Internacional del Trabajo, ¿Qué sabemos sobre los programas y politicas de Primer Empleo en América Latina?, 26.

76 Ibíd.

77 Art. 195.1 y ss. Del CT. 
art. 26 del CT, implementó el contrato de trabajo juvenil, con el objeto de promover el acceso al primer empleo de personas entre dieciocho y veintiséis años de edad, de preferencia sin experiencia laboral previa, por lo menos no en el mercado laboral formal ${ }^{78}$. Esta preferencia, no obstante, no consta en la norma, sin embargo cabe esa posibilidad de interpretación que podría ser incorporada, aunque de forma ilegal, en la regulación secundaria que haga el Ministerio del Trabajo ${ }^{79}$.

Mediante esta modalidad de contrato, y siempre que la contratación del trabajador joven sea superior a un año ${ }^{80}$ y no implique sustitución de otro trabajador indefinido de la plantilla, el Estado asume el pago del aporte patronal a la Seguridad Social, aunque con condiciones adicionales: que la remuneración fijada para el contrato juvenil no supere los dos salarios básicos

78 Según el último inciso del art. 3 del Acuerdo Ministerial MDT-20160158, del Ministerio del Trabajo, que contiene la normativa para la aplicación de la Ley, serán considerados para el beneficio de la exención del pago a la seguridad social los trabajadores que no hubieren laborado más de seis meses continuos en relación de dependencia a jornada completa.

Efectivamente, como pasó con la figura del contrato de trabajo por obra o servicio determinado dentro del giro del negocio, en que el Ministerio del Trabajo limitó lo previsto en la ley reformatoria al CT, y reguló más allá de lo que jurídicamente debía hacer, en este caso podría darse la misma circunstancia. Sobre el análisis descrito, consultar: Lanas Medina, Elisa, «Reformas al Código del Trabajo introducidas por la Ley de Justicia Laboral y Reconocimiento del Trabajo en el Hogar.», Revista de Derecho FORO No. 23 (II semestre de 2015) en prensa.

Después de la reforma introducida por la Ley de Justicia Laboral y reconocimiento del Trabajo en el Hogar al CT, generalmente debería ser un contrato indefinido, y excepcionalmente un contrato por obra o servicio determinado dentro del giro del negocio por más de un año. 
unificados, y que el porcentaje de estos contratos no supere el 20 $\%$ del total de nómina estable de trabajadores en cada empresa. En caso de que la remuneración supere los dos salarios básicos unificados, el empleador deberá asumir el aporte patronal por el salario que supere ese valor; así mismo, si el porcentaje de contratados bajo esa modalidad supera el $20 \%$, será el empleador el que deba pagar el total del aporte patronal de esos trabajadores que superen el porcentaje.

El Acuerdo Ministerial MDT-2016-0158, del Ministerio del Trabajo, que contiene la normativa para la aplicación de la Ley, prevé porcentajes mínimos de contratación obligatoria, bajo esta modalidad, en función de la actividad económica, para las empresas que cuenten con más cincuenta trabajadores, siendo voluntaria la aplicación de este contrato para las empresas o empleadores con menos trabajadores.

Como se adelantó anteriormente, este tipo de medidas, cuando tienen un carácter temporal y bajo ciertos parámetros, pueden ser afectivas para incrementar los porcentajes de empleo adecuado de personas jóvenes. No obstante, la aplicación de un límite a la remuneración de dos salarios mínimos, seguramente va a incentivar contrataciones de trabajadores en las escalas remunerativas más bajas. Asimismo, la aplicación de estos contratos puede desplazar la contratación de otros colectivos con dificultad de acceso a trabajos adecuados, como mujeres, desempleados de larga duración, mayores de cuarenta y cinco años, entre otros.

En definitiva, se observa lo parcial e inmediatista de las medidas de fomento de empleo, que miran únicamente al horizonte cercano y coyuntural de una realidad que es estructural y es la de 
la difícil situación del empleo en Ecuador.

Es necesario planificar una política integral de empleo en el país, que pase por mejorar los niveles de empleabilidad de la población y no perjudique las condiciones laborales de los que están ocupados.

\section{CONCLUSIONES}

El país ha implementado en los últimos años políticas públicas de empleo, por vía de varias reformas asistemáticas y desordenadas, que han respondido a la coyuntura más que a una planificación clara y de largo alcance.

Dentro de dichos mecanismos, se encuentra la política pasiva prevista en el seguro de desempleo. El alcance de esta medida, sin embargo, debería ser mayor, a efectos de que no sólo asegure una renta mínima al beneficiario por cinco meses, sino que constituya un verdadero apoyo al desempleado para que aproveche el tiempo de paro para mejorar sus habilidades y destrezas, a efectos de volver a reinsertarse en el mercado laboral de forma satisfactoria.

En lo que respecta a las políticas activas de empleo, si bien existe un servicio nacional de colocaciones, siendo este un importante mecanismo que permite superar las desigualdades provocadas por la falta de información entre oferentes y demandantes de trabajo; no obstante, es necesario lograr una colocación más dinámica, para lo que hay que conocer mejor las necesidades de los potenciales demandantes del servicio. Lo deseable, sin 
embargo, es avanzar hacia la consideración de la colocación laboral como un derecho, al que deberían acceder todas las personas en aptitud de trabajar, que sea disponible y de fácil acceso, que forme parte de un sistema integrado de formación capacitación - colocación.

En relación a la capacitación laboral, Ecuador registra una evolución significativa, no obstante, una vez institucionalizada la misma, hay que mejorar la oferta de capacitación en sintonía con las iniciativas internacionales, orientadas a la formación técnica o capacitación laboral, que sugieren enfatizar el desarrollo de habilidades suaves y habilidades para la vida.

Por otro lado, mientras en otros entornos se evalúa la posibilidad de reducir la jornada laboral, sin afectación a la remuneración, o de cambiar la lógica de recompensa del trabajo, que no mida primordialmente el tiempo de dedicación del trabajador, parece una involución que en Ecuador se planteen medidas como la reducción impuesta de jornada y remuneración; o la de prolongar la jornada laboral diaria con el único ánimo de abaratar al empleador el costo del trabajo prolongado más allá de los límites legales, sociales y psicológicos admitidos.

Finalmente, respecto de la política activa de empleo juvenil mediante subvenciones a la contratación, este tipo de medidas, cuando tienen un carácter temporal y bajo ciertos parámetros, pueden ser afectivas para incrementar los porcentajes de empleo adecuado de personas jóvenes. No obstante, la aplicación de un límite a la remuneración de dos salarios mínimos, seguramente va a incentivar contrataciones de trabajadores en las escalas remunerativas más bajas. Asimismo, la aplicación de estos contratos puede desplazar la contratación de otros colectivos 
con dificultad de acceso a trabajos adecuados, como mujeres, desempleados de larga duración, mayores de cuarenta y cinco años, entre otros.

En definitiva, se observa lo parcial e inmediatista de las medidas de fomento de empleo, que miran únicamente al horizonte cercano y coyuntural de una realidad que es estructural y es la de la difícil situación del empleo en Ecuador.

Para mejorar de forma efectiva los niveles de empleabilidad en el país, se hace imperioso tener en consideración la evolución de las distintas categorías de empleo, con desagregación por lo menos de edad y género, lo que permitirá tener un panorama muy claro de los colectivos que tienen mayores dificultades de acceso a un trabajo adecuado en el país. A partir de allí, y aplicando lo previsto en la Constitución que nos rige, hay que diseñar un sistema de fomento de empleo en el que se planteen políticas integrales de acceso a un trabajo digno, sea este subordinado, autoempleo o en asociatividad. 


\section{BIBLIOGRAFÍA}

CABEZA PEREIRO, Jaime, y Cardona Rubert, María B. «Políticas de empleo (I)». En Políticas sociolaborales. Tratados y manuales. Navarra: Thomson Reuters, 2014.

CAMAS RODA, Ferrán, y Rodríguez-Piñero Royo, Miguel. «Políticas de empleo (II): políticas activas». En Políticas Sociolaborales. Tratados y manuales. Navarra: Thomson Reuters, 2014.

CEPAL, OIT. «Coyuntura Laboral en América Latina y el Caribe. La evolución del empleo en las empresas de menor tamaño entre 2003 y 2013: mejoras y desafíos», octubre de 2015.

Chacartegui Jávega, Consuelo y Valle Muñoz, Francisco. «Políticas de flexibilidad». En Políticas Sociolaborales. Un enfoque multidisciplinar. Economía y Empresa, No. 17. Barcelona: UOC, 2004.

JARA IÑIGUEZ, Irma. «Cómo deben ser consideradas las características y limitaciones locales en las teorías de construcción de las políticas públicas. Estudio de las políticas de capacitación en el Ecuador: 1978-2011». Universidad Andina Simón Bolívar, Sede Ecuador, 2015. 
LANAS MEDINA, Elisa. «Reformas al Código del Trabajo introducidas por la Ley de Justicia Laboral y Reconocimiento del Trabajo en el Hogar.» Revista de Derecho FORO No. 23 (II semestre de 2015).

LARRUE, Corinne, Knoepfel, Peter, y otros. «Hacia un modelo de análisis de políticas públicas operativo. Un enfoque basado en los actores, sus recursos y las instituciones». Ciencia Política, Perspectivas teóricas, No 3 (junio de 2007).

LÓPEZ GANDÍA, Juan. «Las políticas pasivas: protección por desempleo». En Políticas sociolaborales. Tratados y manuales. Navarra: Thomson Reuters, 2014.

MONESTEROLO, Graciela. «Jornadas laborales prolongadas y sus repercusiones en el buen vivir del trabajador y de su familia». Revista de Derecho FORO 19 (I semestre de 2013): 61-77.

MORENO GENÉ, Josep. «La nueva regulación de las Prácticas Académicas Externas de los estudiantes universitarios: la potenciación de la finalidad formativa y de mejora de la empleabilidad». Revista de Trabajo y Seguridad Social No. 350 (2012).

OFICINA INTERNACIONAL DEL TRABAJO, Tendencias mundiales del empleo juvenil 2015 Promover la inversión en empleos decentes para los jóvenes. Ginebra, 2015.

ORGANIZACIÓN INTERNACIONAL DEL TRABAJO. ¿Qué sabemos sobre los programas y políticas de Primer Empleo 
en América Latina? Juventud e informalidad. Perú: Oficina Regional para América Latina y el Caribe, 2015.

ORGANIZACIÓN INTERNACIONAL DEL TRABAJO «Servicios públicos de empleo en América Latina y el Caribe. Ecuador, características principales y desafíos». , 2015.

PRADOS DE REYES, Francisco. «Modalidades contractuales y política de empleo juvenil». En Retos del Derecho del Trabajo frente al desempleo juvenil. Monografías de temas laborales 52. Granada: Consejo Andaluz de Relaciones Laborales, 2014.

RAMOS MARTÍN, Francisco. «Políticas de empleo». En Políticas sociolaborales. Economía y Empresa 17. Barcelona: Editorial UOC, 2004.

RUESGA Benito, Santos y otros. Economía del trabajo y política laboral. $2^{\mathrm{a}}$ edición. Economía y Empresa. Pirámide, 2014.

SÁNCHEZ-RODAS, Cristina. «Formas no laborales de inserción de los jóvenes en el marcado de trabajo». En Retos del Derecho del Trabajo frente al desempleo juvenil. Monografías de temas laborales 52. Granada: Consejo Andaluz de Relaciones Laborales, 2014.

WELLER, Jürgen. «La inserción laboral de los jóvenes: tensiones y perspectivas». En Mundos del trabajo y políticas públicas en América Latina. Quito: FLACSO Ecuador y Ministerio de Cultura, 2008. 